\title{
Personalidad y perfiles clínicos relacionados con el éxito o recaída en el tratamiento de jugadores patológicos
}

\section{Personality and clinical profiles related to rehabilitation or relapse in the treatment of pathological gamblers}

\author{
Margarita Ortiz-Tallo ${ }^{1}$, María J. Blanca ${ }^{1}$, Marta Ferragut ${ }^{1}$, Sebastián Cobos ${ }^{2}$ \\ ${ }^{1}$ Facultad de Psicología. Universidad de Málaga, España. \\ ${ }^{2}$ Colaborador Grupo Investigación HUM 300, España.
}

Disponible online 30 de abril de 2011

\begin{abstract}
El objetivo del presente estudio es conocer si existen diferencias en patrones de personalidad y síndromes clínicos en función de la rehabilitación o recaída en el juego patológico. Para ello, se ha llevado a cabo un seguimiento a 176 jugadores de un centro de rehabilitación cuya terapia está centrada en grupos de autoayuda de dos años de duración, dividida en tres fases de ocho meses cada una. La rehabilitación se consigue con la finalización de las tres fases, considerándose recaída inmediata el abandono en la primera, o tardía si el abandono es en la segunda fase. Se realizó una evaluación previa al tratamiento con el Inventario Clínico Multiaxial de Millon. Se analizan las diferencias en patrones de personalidad y síndromes clínicos entre los participantes que recaen en una de las fases y los que alcanzan la rehabilitación. El 61,93\% de los participantes alcanzan el éxito terapéutico. Los resultados muestran que sólo la personalidad antisocial presenta diferencias significativas, presentando menor puntuación los participantes que finalizan el tratamiento. Por otro lado, no se encontraron diferencias en el número de personas que se rehabilitan en función de su perfil clínico previo.
\end{abstract}

Palabras Clave: juego patológico, personalidad, antisocial, rehabilitación.

The goal of the present study was to determine whether there are differences between pathological gamblers in personality patterns and clinical syndromes as a function of rehabilitation or relapse. We followed up 176 gamblers from a rehabilitation centre who underwent therapy in self-help groups. Therapy lasted 2 years and was divided into three 8-month phases. Individuals who dropped out in the first phase were considered to have relapsed; those who dropped out in the second phase were considered to be cases of delayed relapse; those who stayed until the end of phase three were considered rehabilitated. Participants were assessed using the Millon Multiaxial Clinical Inventory prior to treatment. We analyzed the differences in personality patterns and clinical syndromes between participants who relapsed in the first or second phase and those who achieved rehabilitation. In total, $61.93 \%$ of the participants achieved therapeutic success. The only significant difference was in the antisocial personality variable; participants who completed the treatment programme had lower scores in this variable. No differences were found in the number of people who were rehabilitated as a function of their previous clinical profile.

Keywords: pathological gambling, personality, antisocial, rehabilitation.

Expresamos nuestro agradecimiento a AMALAJER, Asociación Malagueña de Jugadores en Rehabilitación, y a su presidente Francisco Abad, que ha facilitado todo lo necesario para llevar a cabo este estudio y a todas aquellas personas que sufren como consecuencia del juego patológico y que han prestado su importante colaboración.

Correspondencia a: Margarita Ortiz-Tallo, Facultad de Psicología. Campus de Teatinos s/n. 29071 - Málaga. Telf.: 952132555. Fax: 952131101 E-mail: mortiztallo@uma.es. Correo electrónico de los autores: mortiztallo@uma.es, blamen@uma.es, mferragut@uma.es, smcobos@andaluciajunta.es 
El juego patológico es frecuentemente asociado a la existencia de un diagnóstico dual en el que coexisten alteraciones de la personalidad y sintomatología clínica diversa (Echeburúa y Fernández-Montalvo, 2008; Kerber, Black y Buckwalter, 2008; Petry, Stinson y Grant, 2005). Una de las explicaciones de esta alta comorbilidad está relacionada con los rasgos de personalidad previos que pueden predisponer a las personas a determinada sintomatología clínica. La mayoría de las investigaciones han evaluado rasgos concretos de personalidad, encontrando altos niveles de impulsividad y de búsqueda de sensaciones en los jugadores patológicos (Blaszczynski, Steel, y McConaghy, 1997; Castellani y Rugle, 1995; Petry, 2000). Sin embargo, estudios recientes evalúan la comorbilidad del juego patológico con trastornos de personalidad que ya suponen un conjunto de rasgos, emociones, sentimientos y comportamientos (Alvarez-Moya et al., 2010; Bagby, Vachon, Bulmash y Quilty, 2008; Bahamón, 2006; Fernández-Montalvo y Echeburúa, 2004; Ortiz-Tallo, Cancino y Cobos, en prensa; Pietrzafc y Petry, 2005).

La evidencia empírica sugiere la existencia en los jugadores patológicos de distintos perfiles clínicos, en los que coexisten varios patrones de personalidad patológicos y síndromes clínicos asociados (Blaszczynski y Nower 2002; CunninghamWilliams y Hong 2007; Durdle y Stewart, 2007; Iancu, Lowengrub, Dembinsky, Kotler, y Dannon, 2008; Ledgerwood y Petry 2006; Sacco, Cunningham-Williams, Ostmann, Spitznagel, 2007). En este sentido, Blaszczynski y Nower (2002) identifican tres perfiles, el primero de ellos con problemas de conducta, el segundo de vulnerabilidad emocional y el tercero con un perfil antisocial. En España, Alvarez-Moya et al. (2010) definen cuatro perfiles: el desorganizado e inestable, con alta impulsividad, rasgos de personalidad patológicos, abuso de alcohol y drogas; el esquizoide, con evitación emocional y abuso de alcohol; el de búsqueda de sensaciones e impulsividad, sin patología asociada; y el que refleja buen funcionamiento o normalidad, que constituiría un perfil de personalidad general adaptado y sin alteraciones psicopatológicas. Por su parte, Ortiz-Tallo, Cancino y Cobos (en prensa) encuentran una mayor frecuencia entre jugadores patológicos de los trastornos y rasgos clínicos de personalidad dependiente, obsesivocompulsivo, antisocial y narcisista. A su vez, encuentran dos perfiles clínicos que se han denominados "obsesivo-compulsivo" y "antisocial". El primero presenta puntuaciones altas en patrones de menor gravedad como obsesivo-compulsivo, pasivo-agresivo y dependiente, y con ausencia de síndromes clínicos asociados. El segundo, con mayor patología, presenta puntuaciones más altas que el anterior en los patrones de personalidad, pero principalmente en antisocial, pasivo-agresivo, autodestructivo, paranoide, agresivo-sádico, narcisista, límite y esquizotípico, a su vez que puntuaciones más altas en todos los síndromes clínicos, siendo mayores en los síndromes de abuso de drogas, alcohol, trastorno delirante y pensamiento psicótico.
En general en todos los estudios, el perfil de mayor frecuencia del jugador es el de varón, con rasgos de personalidad patológicos o trastorno de personalidad, siendo el antisocial uno de trastornos encontrados de forma reiterada. Los síndromes clínicos son variados, estando presente en la mayoría el abuso de alcohol y/o drogas (Blaszczynski y Nower, 2002; Janiri, Martinotti, Dario, Schifano y Bria, 2007; Jiménez-Murcia et al., 2010).

La identificación de subgrupos homogéneos en jugadores patológicos con determinados perfiles clínicos podría ser importante para el diseño de las estrategias terapéuticas adecuadas. Asimismo, conocer si hay perfiles que predicen el éxito en la terapia o una mayor probabilidad de recaída ayudaría a la toma de decisiones terapéuticas del proceso de rehabilitación.

La investigación ha mostrado la existencia de tratamientos eficaces en jugadores patológicos, como las intervenciones cognitivo-conductuales que han demostrando su efectividad a corto y largo plazo (Blaszczynski, 2005; Gooding y Tarrier, 2009; Jiménez-Murcia et al., 2007; Ladouceur, Sylvain, Boutin, Lachance, Doucet y Leblond, 2003; Pallesen, Mitsem, Kvale, Johnsen, y Molde, 2005). Cunninngham-Williams et al. (2005) hallaron que algunos rasgos de personalidad como búsqueda de sensaciones, baja capacidad de auto-dirección y de cooperación serían indicadores de riesgo de recaída. En esta línea, consideramos de interés profundizar si existen perfiles clínicos que estén relacionados con una mayor probabilidad de éxito o de recaída del tratamiento. Por otro lado, es difícil encontrar investigaciones científicas en tratamientos de autoayuda o apoyo mutuo llevadas a cabo en organizaciones de jugadores patológicos.

El objetivo del presente estudio, siguiendo el modelo de Millon (1976, 1998), es conocer si existe asociación entre los patrones de personalidad y síndromes clínicos con la posibilidad de sufrir recaídas a lo largo de un proceso de tratamiento en el juego patológico. Para ello, se ha llevado a cabo un seguimiento a un grupo de jugadores de un centro de rehabilitación cuya terapia está centrada en grupos de autoayuda de dos años de duración, dividida en tres fases de ocho meses cada una. Asimismo, como continuación del citado estudio de OrtizTallo, Cancino y Cobos (en prensa), los participantes fueron divididos según el perfil clínico que presentaban inicialmente, "obsesivo-compulsivo" o "antisocial", con objeto de averiguar si mostraban distinta distribución en la permanencia en el tratamiento. Es decir, el segundo objetivo es averiguar si los jugadores que consiguen la rehabilitación dos años después presentan distinto perfil clínico inicial que los que recaen, bien en la primera o segunda fase del tratamiento. Es de esperar que el perfil "obsesivo-compulsivo", dada su menor patología de personalidad tenga mejor pronóstico terapéutico que el "perfil antisocial". 
Método

\section{Participantes}

Participaron 176 hombres que acudieron a tratamiento a la Asociación Malagueña de Jugadores en Rehabilitación (AMALAJER) de Málaga, con edades comprendidas entre 20 y 72 años $(\mathrm{M}=41,48 ; \mathrm{DT}=12,11)$.

Los criterios de inclusión han sido: 1) haber acudido a la citada Asociación para solicitar ayuda durante los años 2006 al 2008; 2) tener un diagnóstico de juego patológico valorado por el psicólogo del centro; 3 ) haber sido evaluado dentro de los 3 primeros meses de haber acudido a la Asociación; 4) ser varón; 5) haber cumplimentado de forma correcta el cuestionario MCMI-II y 6) tener datos sobre su éxito o abandono terapéutico dos años después. Los criterios de exclusión han sido: 1) ser mujer, por ser muy escaso el número que las representaba; 2) presentar perfiles inválidos en el MCMI-II, según los índices de sinceridad, deseabilidad y alteración y 3 ) no tener datos fiables sobre su evolución posterior.

El tiempo de terapia, dividido en tres fases de 8 meses cada una, que recibieron los participantes estuvo comprendido entre 2 y 24 meses, con una media de 18,51 (DT =7,96). Alcanzaron la primera fase 38 sujetos, la segunda 29 y la tercera, con finalización y éxito de la misma, 109 participantes. Así, el 61,93\% de los varones que se han incluido en este estudio consiguieron finalizar con éxito la terapia. En la tabla 1 se muestran las características de la muestra por cada fase de tratamiento alcanzada. No se hallan diferencias en función del estado civil, nivel de estudios, situación laboral ni tiempo de juego. Sí se encuentran diferencias en función de la edad, siendo menor la edad de los que alcanzan el éxito terapéutico.

Tabla 1. Medias y desviación típica entre paréntesis de la edad y el tiempo de juego en función de las fases alcanzadas de tratamiento, estadístico $F$ y probabilidad asociada. Número de participantes en función del estado civil, estudios y situación laboral, estadístico $\chi^{2}$ y probabilidad asociada.

\begin{tabular}{|c|c|c|c|c|c|}
\hline & $\begin{array}{c}1^{\mathrm{a}} \text { Fase } \\
(8 \text { meses })\end{array}$ & $\begin{array}{c}2^{\mathrm{a}} \text { Fase } \\
(16 \text { meses })\end{array}$ & $\begin{array}{c}3^{\mathrm{a}} \text { Fase } \\
(24 \text { meses })\end{array}$ & $F / \chi^{2}$ & $p$ \\
\hline Edad & $\begin{array}{c}45,61 \\
(13,55)\end{array}$ & $\begin{array}{c}41,52 \\
(13,21)\end{array}$ & $\begin{array}{c}40,04 \\
(11,02)\end{array}$ & 3,05 & 0,05 \\
\hline Estado civil & & & & 5,06 & 0,28 \\
\hline Soltero & 9 & 11 & 33 & & \\
\hline Casado & 22 & 17 & 66 & & \\
\hline Divorciado & 7 & 1 & 10 & & \\
\hline Estudios & & & & 4,16 & 0,66 \\
\hline Sin estudios finalizados & 1 & - & 4 & & \\
\hline Primaria & 19 & 19 & 67 & & \\
\hline Secundaria & 13 & 6 & 29 & & \\
\hline Universitarios & 5 & 4 & 9 & & \\
\hline Situación laboral & & & & 1,97 & 0,76 \\
\hline Activo & 35 & 27 & 102 & & \\
\hline Desempleo & 0 & 1 & 2 & & \\
\hline Jubilación & 3 & 1 & 5 & & \\
\hline Total & 38 & 29 & 109 & & \\
\hline Tiempo de juego & $\begin{array}{c}10,74 \\
(10,89) \\
\end{array}$ & $\begin{array}{l}11,79 \\
(8,99) \\
\end{array}$ & $\begin{array}{c}9,67 \\
(8,45) \\
\end{array}$ & 0,69 & 0,50 \\
\hline
\end{tabular}

Material

Para la recogida de los datos sociodemográficos se administró un cuestionario a los participantes que completaron al inicio de la primera entrevista en la Asociación, incluyendo el estado civil, edad, nivel de estudios, situación laboral y tiempo de juego.

Para analizar los patrones de personalidad y los síndromes clínicos se utilizó el Inventario Clínico Multiaxial de Millon (MCMI-II) (Millon, 1999). Se ha administrado esta versión en vez de la adaptada actualmente en España por ser la utilizada en el momento inicial por los primeros participantes evaluados.

Este inventario autoinformado evalúa personalidad y síndromes clínicos a través de 175 ítems de respuesta dicotómica, verdadero o falso. Tiene tres escalas de validez: sinceridad, deseabilidad y alteración, diez escalas básicas de personalidad: esquizoide, evitativa, dependiente, histriónica, narcisista, antisocial, agresivo-sádica, compulsiva, pasivo-agresiva y autodestructiva, y tres escalas de personalidad de mayor gravedad: esquizotípica, límite y paranoide. En relación con los síndromes clínicos, considerados más temporales, evalúa ansiedad, histeriforme, hipomanía, distimia, abuso de alcohol, abuso de drogas. $\mathrm{y}$, de mayor gravedad, pensamiento psicótico, depresión mayor y trastorno delirante. Se considera que una puntación de Tasa Base (TB) mayor que 60 indica presencia de rasgos clínicos de personalidad y de sintomatología clínica asociada, y mayor que 75 indicaría la presencia de patología. En la validación española el instrumento presenta criterios adecuados de fiabilidad y validez. El coeficiente de consistencia interna medio es de 0,78 para las escalas de personalidad y de 0,80 para las escalas de síndromes clínicos (Ávila-Espada y Jiménez-Gómez, 1999).

\section{Procedimiento}

Un psicólogo de la Asociación realizó la evaluación inicial de forma individual en todos los participantes, cumplimentando los datos sociodemográficos en la primera sesión. En sucesivas sesiones, se recogió la historia clínica general y se administró el MCMI-II. Durante los dos años siguientes del inicio de la terapia, se evaluó la evolución de todos los participantes, registrándose el tiempo de tratamiento y si superaba o no las distintas fases del mismo.

La terapia de la Asociación se basa en grupos de autoayuda con jugadores que acuden a rehabilitación y jugadores rehabilitados. Está dividida en tres fases, con una duración aproximada de ocho meses cada una. Cada fase tiene unos objetivos específicos y diferenciados. La Fase 1 tiene como objetivo el control estimular relacionado con la conducta de juego, así deben dejar de jugar, de beber, de acudir a lugares de riesgo y/o visitar amistades de riesgo; se les impide manejar su economía teniendo que ser un familiar el que se ocupe de este aspecto. En la siguiente fase, el objetivo principal es trabajar las actitudes (impulsividad, prepotencia, diálogo, etc.). Finalmente, la ter- 
cera fase se ocupa de la relación de pareja y de las relaciones familiares. En la primera y segunda fase acuden a terapia de grupo de dos a tres días en semana, coordinada por un jugador rehabilitado. Las sesiones individuales con el psicólogo son una vez al mes. Paralelamente, un miembro de la familia también recibe sesiones de terapia con un grupo de familiares de jugadores rehabilitados.

El alta terapéutica, con éxito total del tratamiento, se consigue al superar las tres fases de las que consta el programa. Por tanto, se considera que han conseguido la rehabilitación aquellos que han finalizado las tres fases, mientras que se considera que han recaído de forma inmediata los que la abandonan en la primera o, de forma tardía, los que abandonan en la segunda fase.

\section{Análisis estadístico}

Con el objetivo de analizar si existen diferencias en las puntuaciones TB de las escalas y síndromes clínicos de personalidad entre los participantes que llegan a las primera, segunda o tercera fase del tratamiento, se ha realizado un análisis de covarianza (ANCOVA), considerando la edad como covariable dado que se hallaron diferencias significativas entre las tres fases en esta variable (tabla 1). En todos los casos, se ha satisfecho el supuesto de homogeneidad de las pendientes de regresión, así como la normalidad y homogeneidad de los residuales.
Por otro lado, se ha realizado una prueba $\chi^{2}$ con objeto de analizar si los participantes clasificados según los dos perfiles de personalidad, perfil "obsesivo-compulsivo" (compuesto por las escalas obsesivo-compulsivo, pasivo-agresivo y dependiente) y "perfil antisocial" (compuesto por las escalas antisocial, pasivo-agresivo, autodestructivo, paranoide, agresivo-sádico, narcisista, límite y esquizotípico), encontrados por Ortiz-Tallo, Cancino y Cobos (en prensa) muestran distinta distribución en la permanencia en el tratamiento.

\section{Resultados}

Los resultados del ANCOVA con las distintas fases de tratamiento alcanzadas como factor y la edad como covariable, y las escalas y síndromes clínicos de personalidad como variables dependientes se presentan en la tabla 2. Los resultados muestran sólo diferencias significativas en la escala antisocial, presentando menor puntuación los participantes que finalizan el tratamiento y llegan a la tercera fase.

Los resultados de la prueba $\chi^{2}$ con las fases de tratamiento y los dos perfiles de personalidad, muestra que ambas variables son independientes $\left[\chi^{2}(2, N=176)=2,16, p=0,34\right]$, existiendo la misma tendencia en la distribución de porcentajes en los mencionados perfiles. Los resultados se presentan en la tabla 3 .

Tabla 2. Medias ajustadas a la edad de las escalas de personalidad y síndromes clínicos del MCMI-II en función de la fase alcanzada de tratamiento, estadístico $F$, probabilidad asociada y eta cuadrado parcial.

\begin{tabular}{|c|c|c|c|c|c|c|}
\hline Escalas y síndromes clínicos & $\begin{array}{c}1^{\mathrm{a}} \text { Fase } \\
(8 \mathrm{meses})\end{array}$ & $\begin{array}{c}2^{\mathrm{a}} \text { Fase } \\
(16 \text { meses })\end{array}$ & $\begin{array}{c}3^{\mathrm{a}} \text { Fase } \\
(24 \text { meses })\end{array}$ & $\mathrm{F}(2,172)$ & $p$ & $\eta_{\text {parcial }}^{2}$ \\
\hline Esquizoide & 61,76 & 61,27 & 57,29 & 0,80 & 0,45 & 01 \\
\hline Fóbico & 50,21 & 52,31 & 42,90 & 1,64 & 0,20 &, 02 \\
\hline Dependiente & 67,15 & 62,71 & 60,36 & 1,05 & 0,35 &, 01 \\
\hline Histriónico & 59,52 & 53,18 & 52,92 & 1,04 & 0,36 &, 01 \\
\hline Narcisista & 54,61 & 57,29 & 49,50 & 1,28 & 0,28 & ,02 \\
\hline Antisocial & 57,62 & 63,99 & 49,46 & 4,12 & 0,02 &, 05 \\
\hline Agresivo-Sádico & 48,01 & 53,22 & 49,43 & 0,31 & 0,73 &, 004 \\
\hline Compulsivo & 57,18 & 70,12 & 66,45 & 2,67 & 0,07 & 03 \\
\hline Pasivo-Agresivo & 47,35 & 46,01 & 46,43 & 0,02 & 0,98 & $<, 001$ \\
\hline Autodestructivo & 58,49 & 55,69 & 51,63 & 0,93 & 0,40 &, 01 \\
\hline Esquizotípico & 48,76 & 54,51 & 44,92 & 1,95 & 0,15 &, 02 \\
\hline Límite & 46,01 & 44,66 & 42,61 & 0,24 & 0,79 &, 003 \\
\hline Paranoide & 60,31 & 65,21 & 57,64 & 1,55 & 0,22 & ,02 \\
\hline Ansiedad & 50,31 & 43,13 & 39,59 & 1,75 & 0,18 &, 02 \\
\hline Histeriforme & 46,05 & 39,99 & 40,92 & 0,58 & 0,56 &, 01 \\
\hline Hipomanía & 51,41 & 49,31 & 50,39 & 0,07 & 0,93 &, 001 \\
\hline Neurosis Depresiva & 37,49 & 34,91 & 32,05 & 0,53 & 0,59 & 01 \\
\hline Abuso Alcohol & 64,61 & 59,25 & 58,93 & 0,90 & 0,41 &, 01 \\
\hline Abuso Drogas & 52,66 & 53,56 & 47,512 & 1,15 & 0,32 &, 01 \\
\hline Pensamiento Psicótico & 52,46 & 50,24 & 47,11 & 0,63 & 0,53 &, 01 \\
\hline Depresión Mayor & 47,68 & 40,24 & 38,10 & 1,71 & 0,18 &, 02 \\
\hline Trastorno Delirante & 59,53 & 66,86 & 58,44 & 2,20 & 0,11 &, 03 \\
\hline
\end{tabular}


Tabla 3. Número $(n)$ y porcentaje de participantes de los perfiles clínicos "obsesivo-compulsivo" y "antisocial" en función de la fase alcanzada de tratamiento.

\begin{tabular}{llccc}
\hline Perfiles & & $\begin{array}{c}1^{\mathrm{a}} \text { Fase } \\
(8 \mathrm{meses})\end{array}$ & $\begin{array}{c}2^{\mathrm{a}} \text { Fase } \\
(16 \text { meses })\end{array}$ & $\begin{array}{c}3^{\mathrm{a}} \text { Fase } \\
(24 \mathrm{meses})\end{array}$ \\
\hline Obsesivo-compulsivo & $n$ & 14 & 11 & 53 \\
& Porcentaje & 17,9 & 14,1 & 67,9 \\
Antisocial & $n$ & 24 & 18 & 56 \\
& Porcentaje & 24,5 & 18,4 & 57,1 \\
\hline
\end{tabular}

Discusión

El objetivo del presente estudio era conocer si existía asociación entre los patrones de personalidad y los síndromes clínicos con la posibilidad de sufrir recaídas a lo largo de un proceso de tratamiento en el juego patológico. Igualmente, se pretendía averiguar si los jugadores que conseguían la rehabilitación presentaban distinto perfil clínico inicial que los que recaían en las distintas fases de la terapia. Para ello se llevó a cabo un seguimiento de dos años a un grupo de jugadores que fueron evaluados al llegar a un centro de rehabilitación que centra la terapia en los grupos de autoayuda dividida en tres fases de ocho meses de duración.

En relación con las diferencias en patrones de personalidad y síndromes clínicos según la fase del tratamiento alcanzado sólo se han encontrado diferencias significativas en una de las escalas de personalidad, la antisocial. Así, los jugadores patológicos que consiguen finalizar la terapia y la rehabilitación son aquellos que han puntuado más bajo en la escala antisocial de personalidad. Por tanto, podría haber mayor probabilidad de éxito en la terapia en aquellas personas que su personalidad no sea antisocial caracterizada por alto conflicto con las normas sociales, alta impulsividad y graves problemas de comportamiento tales como robos, mentiras, peleas o crueldad (Millon y Davis, 2001).

Este hallazgo se podría relacionar con estudios que proponen que los jugadores patológicos que más dificultades tienen para la rehabilitación son aquellos que más impulsividad presentan como rasgo de personalidad (Blaszczynski, Steel, y McConaghy, 1997; Petry 2000). Asimismo, Bahamón (2006) indica que los jugadores con personalidad antisocial no suelen buscar ayuda y tratamiento para sus problemas con el juego, y si lo hicieran tienen menor probabilidad de llevar a cabo la rehabilitación. Por tanto, una puntuación baja en rasgos de personalidad antisocial puede ser un buen predictor para la rehabilitación.

Por otro lado, respecto a si los jugadores que conseguían la rehabilitación presentaban distinto perfil clínico inicial que los que recaían en las distintas fases de la terapia, los resultados muestran que no existen diferencias entre los perfiles "obsesivo-compulsivo" y "antisocial". De esta forma, el perfil antisocial que incluía la coexistencia de patrones de personalidad y síndromes clínicos de gravedad (antisocial, pasivo-agresivo, agresivo-sádico, autodestructivo, esquizotípico, límite, paranoide, abuso de drogas y alcohol, trastorno delirante y pensa- miento psicótico) no presenta de forma significativa un mayor riesgo de abandono o de recaída del proceso de rehabilitación. Este resultado no es acorde con lo esperado, ya que se esperaba encontrar que el perfil "obsesivo-compulsivo", dada su menor patología de personalidad, tuviera mejor pronóstico y mayor éxito en la rehabilitación.

El hecho de no encontrar grandes diferencias entre los jugadores que finalizan y los que no finalizan la terapia puede deberse a la propia efectividad de la terapia, que puede permitir que estructuras de personalidad arraigadas evolucionen hacia unas más saludables. Hay que tener en cuenta que la mayoría de los pacientes, un 61,93\%, completaron el tratamiento, con alta terapéutica y abstinencia del juego tras un periodo de dos años. Otras investigaciones realizadas con tratamientos cognitivo-conductuales, de menor duración, aproximadamente 16 semanas, hallan un porcentaje de éxito parecido al finalizar la terapia, pero con una recaída del 50\% al año y del $70 \%$ dos años después (Cunningham-Williams et al., 2005; Gooding y Tarrier, 2009). Es posible que la terapia basada en grupos de autoayuda facilite la rehabilitación por contener elementos propios de una terapia multimodal: utiliza la figura de un jugador rehabilitado, como referente y como apoyo constante; incluye terapia mensual con el psicólogo; y se realiza tratamiento con la familia que recibe pautas de conductas para reafirmar el proceso de rehabilitación. Además, hay que considerar que esta terapia es de mayor duración que otras terapias cognitivo-conductuales referidas por otros investigadores (Cunningham-Williams et al., 2005; Gooding y Tarrier, 2009; Jiménez-Murcia et al., 2007). La conjunción de terapia multimodal junto con la larga duración, según el modelo de Millon (1976, 1998), podría permitir cambios no sólo en los síndromes clínicos sino también en los patrones de personalidad, facilitando el éxito terapéutico.

En general, los resultados muestran que no existe un patrón de personalidad patológico asociado al éxito o recaída de la terapia basada en grupos de autoayuda. Es posible que existan otros factores que no se hayan considerado en el presente estudio y que sean más relevantes para la rehabilitación, como puede ser la motivación para llevar a cabo el tratamiento o el efecto de la red de apoyo social y familiar. Futuros estudios deberían considerar estas variables, a la vez que evaluar el posible cambio en los patrones de personalidad y síndromes clínicos asociados.

\section{Referencias}

1. Álvarez-Moya, E., Jiménez-Murcia, S., Aymamí, M.A., Gómez-Peña, M., Granero, R., Santamaría, J., Menchón, J.M. y Fernández-Aranda, F. (2010). Subtyping study of a pathological gamblers sample. La Revue Canadienne de Psychiatrie, 55, 498-506.

2. Ávila-Espada, A. y Jiménez Gómez, F. (1999). Adaptación y baremación al español del Inventario Clínico Multiaxial de Millon - II (MCMI-II). Madrid: TEA Ediciones. 
3. Bagby, M., Vachon, D., Bulmash, E y Quilty. L.C. (2008). Personality disorders and pathological gambling: A review and re-examination of prevalence rates. Journal of Personality Disorders, 22, 191-207. doi:10.1521/ pedi.2008.22.2.191

4. Bahamón, M. (2006). Juego patológico: revisión de tema. Revista Colombiana de Psiquiatría, 35, 380-399.

5. Blaszczynski, A. (2005). Pathological gambling: A clinical guide to treatment. Addiction, 100, 565-566. doi:10.1111/ j.1360-0443.2005.01078.x

6. Blaszczynski, A. y Nower, L. (2002) A pathways model of problem and pathological gambling. Addiction, 97, 487-99. doi:10.1046/j.1360-0443.2002.00015.x

7. Blaszczynski, A., Steel, Z. y McConaghy, N. (1997). Impulsivity in pathological gambling: The antisocial impulsivity. Addiction, 92, 75-87. doi:10.1111/j.1360-0443.1997.tb03639.x

8. Castellani, B. y Rugle, L. (1995). A comparison of pathologial gamblers to alcoholics and cocaine misusers on impulsivity, sensation seeking, and craving. The International Journal of the Addictions, 30, 275-289.

9. Cunningham-Williams, R. y Hong, S. (2007). A latent class analysis of problem gambling among a sample of community-recruited gamblers. Journal of Nervous and Mental Disease, 195, 939-947. doi:10.1097/NMD.0b013e31815947e1

10. Cunningham-Williams, R., Grucza, R., Cottler, L., Womack, S., Books, S., Przybeck, T. Spitznagel, E.L. y Cloninger, C.R. (2005). Prevalence and predictors of pathological gambling: results from the St. Louis personality, health and lifestyle (SLPHL) study. Journal Psychiatry Research, 39, 377-390. doi:10.1016/j.jpsychires.2004.09.002

11. Durdle, H. y Stewart, S.H. (2007). Should pathological gambling be considered an obsessive-compulsive spectrum condition?. En J. S. Abramowitz, D. McKay, y S. Taylor (Eds.), Obsessive-compulsive disorder: Subtypes and spectrum conditions (pp. 160-176). New York: Elsevier.

12. Echeburúa, E. y Fernández-Montalvo, J. (2008). Are there more personality disorders in treatment-seeking pathological gamblers than in other kind of patients? A comparative study between the IPDE and the MCMI. International Journal of Clinical and Health Psychology, 8, 53-64.

13. Fernández-Montalvo, J. y Echeburúa, E. (2004). Pathological gambling and personality disorders: An exploratory study with the IPDE. Journal of Personality Disorders, 18, 500-505. doi:10.1521/pedi.18.5.500.51326

14. Gooding, P. y Tarrier, N. (2009). A systematic review and meta-analysis of cognitive-behavioural interventions to reduce problem gambling: Hedging our bets? Behaviour Research and Therapy, 47, 592-607. doi:10.1016/j. brat.2009.04.002

15. Iancu, I., Lowengrub, K., Dembinsky, Y., Kotler, M. y Dannon, P. (2008). Pathological gambling. An update on neuropathophysiology and pharmacotherapy. CNS Drugs,
22, 123-138. doi:10.2165/00023210-200822020-00004

16. Janiri, L., Martinotti, G., Dario, T., Schifano, F. y Bria, P. (2007). The gamblers' Temperament and Character Inventory (TCI) personality profile. Substance Use and Misuse, 42, 975-984. doi:10.1080/10826080701202445

17. Jiménez-Murcia, S., Álvarez-Moya, E.M., Granero, R., Aymamí, M. N., Gómez-Peña, M., Jaurrieta, N., Bibiana, S., Rodriguez-Marti, J. y Vallejo, J. (2007). Cognitive-behavioral group treatment for pathological gambling: Analysis of effectiveness and predictors of therapy outcome. Psychotherapy Research, 17, 544-552. doi:10.1080/10503300601158822

18. Jiménez-Murcia, S. Álvarez-Moya, E., Stinchfield, R., Fernández-Aranda, F., Granero, R., Aymamí, N., Gómez-Peña, M., Jaurrieta, N., Bove, F. y Menchón, J.M. (2010). Age of onset in pathological gambling: Clinical, therapeutic and personality correlates. Journal of Gambling Studies, 26, 235-248. doi:10.1007/s10899-009-9175-3

19. Kerber, C.S., Black, D.W., Buckwalter, K. (2008). Comorbid psychiatric disorders among older adult recovering pathological gamblers. Issues Mental Health Nursing, 29, 1018-1028. doi:10.1080/01612840802274933

20. Ladouceur, R., Sylvain, C., Boutin, C., Lachance, S., Doucet, C. y Leblond, J. (2003). Group therapy for pathological gamblers: a cognitive approach. Behavior Research and Therapy, 41, 587-596. doi:10.1016/S00057967(02)00036-0

21. Lardgerwood, D. y Petry, N. (2006). Psychological experience of gambling and subtypes of pathological gamblers. Psychiatry Research, 144, 17-27. doi:10.1016/j.psychres.2005.08.017

22. Millon, T. (1976). Psicopatología moderna: Enfoque biosocial de los aprendizajes erróneos y de los disfuncionalismos. Barcelona: Salvat.

23. Millon, T. (1998). MCMI-II. Inventario Clínico Multiaxial de Millon II. (Adaptación española de A. Ávila y F. Jiménez). Madrid: TEA Ediciones, S.A.

24. Millon, T. (1999). MCMI-II. Inventario Clínico Multiaxial de Millon. Manual. (2a edicion revisada). Madrid: TEA Ediciones, S.A.

25. Millon, T y Davis, R. (2001). Trastornos de personalidad en la vida moderna. Barcelona: Masson.

26. Ortiz-Tallo, M., Cancino, C. y Cobos, S. (en prensa). Juego patológico, patrones de personalidad y sindromes clínicos. Adicciones.

27. Pallesen, S., Mitsem, M., Kvale, G., Johnsen, B.H. y Molde, H. (2005). Outcome of psychological treatments of pathological gambling: a review and meta-analysis. Addiction, 100, 1412-1422. doi:10.1111/j.1360-0443.2005.01204.x

28. Petry, N.M. (2000). Psychiatric symptoms in problem gambling and non-problem gambling substance abusers. The American Journal on Addictions, 9, 163-171. doi:10.1080/10550490050173235 
29. Petry, N.M, Stinson, F.S. y Grant, B. F. (2005). Comorbidity of DSM-IV pathological gambling and other psychiatric disorders: results from the National Epidemiologic Survey on Alcohol and Related Conditions. Journal of Clinical Psychiatry, 66, 564-574. doi:10.4088/JCP.v66n0504

30. Pietrzak R.H. y Petry, N.M. (2005). Antisocial personality disorder is associated with increased severity of gambling, medical, drug and psychiatric problems among treatmentseeking pathological gamblers. Addiction, 100, 1183-1193. doi:10.1111/j.1360-0443.2005.01151.x

31. Sacco, P., Cunningham-Williams, R., Ostmann, E. y Spitznagel, E. (2007). The association between gambling pathology and personality disorders. Journal of Psychiatric Research, 42, 1122-1130. doi:10.1016/j.jpsychires.2007.11.007

Fecha de recepción: 29 de marzo de 2011 Fecha de recepción de la versión modificada: 5 de abril de 2011

Fecha de aceptación: 12 de abril de 2011 